\title{
On invariant measures for simple branching processes (Summary)
}

\section{E. Seneta}

\begin{abstract}
Problems pertaining to invariant measures of a non-critical Galton-Watson process, whether with or without immigration, may be discussed in terms of measures of a subcritical process with a possibly defective immigration distribution. There is in fact only one such measure satisfying a regular variation condition. This result provides a unifying principle for several contexts of Galton-Watson theory. A full discussion with analytical details will appear elsewhere.
\end{abstract}

\section{The central result}

Let $F(s)$ and $B(s)$ be probability generating functions (p.g.f.'s) on the non-negative integers. We assume that $F(1-)=1$ and $0<F(0)<1$, which implies that $q \in(0,1]$ where $q$ is the smallest root in the interval $[0,1]$ of $F(x)=x$; that $F(s) \neq \alpha+\beta s$ and $m \equiv F^{\prime}(1-)<\infty$; and that $0<B(0)<1$, but $B(1-) \leq 1$.

Our central result pertains to solutions of the form $V(s)=\sum_{i=0}^{\infty} v_{i} s^{i},\left(\left\{v_{i}\right\} \geq\{0\},\left\{v_{i}\right\} \neq\{0\}\right)$ convergent for $s \in[0,1)$ to the functional equation

$$
V(s)=B(s) V(F(s)), \quad s \in[0,1)
$$

where $m<1$. This amounts to an investigation of stationary measures of a subcritical Galton-Watson process, with offspring p.g.f. $F(s)$ and a

Received 20 February 1970. The work was supported by a Nuffield Dominion Travelling Fellowship. Communicated by G.E.H. Reuter . 
possibly defective immigration p.g.f. $B(s)$. The possibility that $B(1-)<I$ is the only novel feature which necessitates a treatment different from [5], Section 5.3; however it is precisely this which enables us to answer not only the open problems in this reference, but also to provide information on the uniqueness of invariant measures for the Galton-Watson process when $m \neq 1$, either with or without immigration. The main result is as follows.

THEOREM. There exists a unique solution to (1) (as usual to a constant multiplier) of the kind we seek satisfying $V(1-x)=x^{-\delta} L(x)$, where $L(x)$ is slowly varying as $x \rightarrow 0+$, and $\delta$ is a finite real number. In fact, $\delta$ is given by $\log B(1-) / \log$, and this solution by

$$
V(s)=c\{1-G(s)\}^{-\delta} V_{1}(s), c=\text { const. }>0 .
$$

Here $G(s)$ is the p.g.f. of the asymptotic conditional distribution of the Galton-Watson process generated by $F(s),[1]$; and $V_{1}(s)$ generates the unique invariant measure for such a process with immigration, with offspring p.g.f. $F(s)$ and (proper) immigration p.g.f. $B(s) / B(1-),[5]$, Section 5.3. Before proceeding to list some of the consequences of this result and the preliminary results involved in its proof, we note that a power-series variant of Karamata's Tauberian Theorem implies that for the solution (2) as $n \rightarrow \infty$

$$
\sum_{i=0}^{n} v_{i} \sim n^{\delta} L(1 / n) / \Gamma(\delta+1) .
$$

\section{Consequences for the ordinary Galton-Watson process}

Kingman [2] has shown that in general quite distinct invariant measures may exist for the Galton-Watson process generated by $F(s)$ with $m \neq 1$. Let $\Pi(s), s \in[0, q)$ generate any such measure. our result implies that there is only one satisfying $V(s) \equiv \exp \Pi(q s)=(1-s)^{-\delta} L(1-s) ;$ in fact $\delta=-1 / \log F^{\prime}(q-)$, etc. A second consequence of similar sort concerns uniqueness of p.g.f. solutions $Q(s)$ to the functional equation $\{1-Q(F(s))\}=m^{\nu}\{1-Q(s)\}$, $s \in[0,1)$, where $m<1$ and $0<v \leq 1$, raised by Rubin and Vere-Jones 
[3]. Our result implies that the only solutions satisfying, for some $\delta$, $Q(s)=1-(1-s)^{1-\delta} L(1-s)$ are given by $Q(s)=1-c(1-G(s))^{\nu}$, $0<c \leq 1$.

A side-product of our work pertains to a random variable $W$ which has the distribution of the limit law of the process when $m<1$, discussed in [1]; or for that of the process when $m>1$, discussed in [4]. In either case, $\int_{0}^{y} \operatorname{Pr}(W>x) d x=L(y)$, where $L(y)$ is slowly varying as $y \rightarrow \infty$. (This implies $\left.E\left(W^{\rho}\right)<\infty, 0<\rho<1.\right)$

\section{Consequences for the Galton-Watson process} with a proper immigration distribution

In the case $m>1$, an invariant measure always exists, but is not in general unique. There is however only one satisfying $V(q s)=(1-s)^{-\delta} L(1-s) ;$ and in fact $\delta=\log B(q-) / \log F^{\prime}(q-)$ etc. (In the case $m<1$, the unique invariant measure always satisfies $V(s)=L(1-s)$.

\section{References}

[1] C.R. Heathcote, E. Seneta and D. Vere-Jones, "A refinement of two theorems in the theory of branching processes", Teor. Verojatnost. i Primenen. 12 (1967), 341-346.

[2] J.F.C. Kingman, "Stationary measures for branching processes", Froc. Amer. Math. Soc. 16 (1965), 245-247.

[3] H. Rubin and D. Vere-Jones, "Domains of attraction for the subcritical Galton-Watson branching process", J. App Z. Probability 5 (1968), 216-219.

[4] E. Seneta, "On recent theorems concerning the supercritical Galton-Watson process", Ann. Math. Statist. 39 (1968), 2098-2102. 
[5] E. Seneta, "Functional equations and the Galton-Watson process", Adv. App 2. Probability 1 (1969), 1-42.

Imperial College,

London,

and

Australian National University,

Canberra, ACT. 